EPJ Web of Conferences 37, 06011 (2012)

DOI: $10.1051 /$ epjconf/20123706011

C) Owned by the authors, published by EDP Sciences, 2012

\title{
Exclusive Central Meson Production in Proton Antiproton Collisions at the Tevatron
}

\author{
Michael Albrow ${ }^{1, \mathrm{a}}$, Artur Swiech ${ }^{2, \mathrm{~b}}$, and Maria Zurek ${ }^{2, \mathrm{c}}$ on behalf of the CDF Collaboration \\ 1 Fermi National Accelerator Laboratory, Batavia, USA \\ 2 Jagiellonian University, Krakow, Poland
}

\begin{abstract}
It has been known since the days of the Intersecting Storage Rings, ISR, at CERN, that one can have $p p$ interactions with more than one pomeron, $\mathbb{P}$, exchanged, known as double pomeron exchange. Exclusive hadronic systems, produced by double pomeron exchange, DPE, have the potential of opening a rich new window on hadron spectroscopy and the diffraction mechanism. We have studied events of the type $p+\bar{p} \rightarrow$ $p+X+\bar{p}$ where $X$ is a hadron pair (mostly $\pi^{+} \pi^{-}$) at $\sqrt{s}=900 \mathrm{GeV}$ and $1960 \mathrm{GeV}$ in the Collider Detector at Fermilab (CDF). The hadron pair is central, $y \approx 0$, and between two rapidity gaps $\Delta y \approx 4$. The dominant process is double pomeron exchange, DPE, with restrictions on the quantum numbers of $\mathrm{X}: \mathrm{Q}=\mathrm{S}=0, \mathrm{C}=+1, \mathrm{~J}=0$ or 2 . The mass spectra, with about $300 \mathrm{~K}$ candidate events assumed to be $\pi^{+} \pi^{-}$, shows strong resonant structures attributed to $f_{0}$ and $f_{2}$ states. We give the ratio of cross sections at $\sqrt{s}=900$ $\mathrm{GeV}$ and $1960 \mathrm{GeV}$, and compare with Regge expectations.
\end{abstract}

\section{Introduction}

The pomeron, $\mathbb{P}$, can be defined as the carrier of 4-momentum between protons when they scatter elastically at high (i.e. collider) energies. It is therefore a strongly interacting color singlet state, at leading order a pair of gluons: $\mathbb{P}=g g$. Of course in QCD it cannot be a pure state, quark pairs and other gluons must evolve in when $Q^{2}$, which we can equate with the 4-momentum $\operatorname{transfer}^{2} t$, becomes large. When $Q^{2}$ is small $\left(\lesssim 2 \mathrm{GeV}^{2}\right)$ which is usually the case with pomeron exchange, perturbative QCD cannot be used to calculate cross sections, as the coupling $\alpha_{s}\left(Q^{2}\right)$ becomes of order 1 . Nonperturbative methods, such as Regge theory, are more applicable (see [1],[2] and [3]). It is a challenge to theorists to derive Regge theory from QCD, but after 40 years it has not happened. Meanwhile the subject is largely data-driven and phenomenological, hence the value of new data such as in this study.

It has been known since times of the ISR, at CERN ( $p p$ with $\sqrt{s}=23-63 \mathrm{GeV}$ ) that one can have $p p$ interactions with more than one pomeron, $\mathbb{P}$, exchanged, known as double pomeron exchange, $D \mathbb{P}$ E. See [4] for a recent review. This process $\mathbb{P}+\mathbb{P} \rightarrow X$ allows an experimental approach to better understand the pomeron. One should not think of the pomerons as isolated entities being emitted from the protons that then interact; the pomeron is only a $t$-channel exchange. In CDF (Collider Detector at Fermilab) we cannot detect the outgoing protons, but it does not matter for this study as long as we can select events with large rapidity gaps $\Delta y \gtrsim 3$ on each side of $X$. In this paper we concentrate on a situation when $M(X) \lesssim 5 \mathrm{GeV} / c^{2}$. Then the interest is for specific ("exclusive") states with well defined quantum numbers.

\footnotetext{
a e-mail: albrow@fnal.gov

b e-mail: artur.swiech@uj.edu.pl

c e-mail: maria.zurek@uj.edu.pl
}

This is an Open Access article distributed under the terms of the Creative Commons Attribution License 2.0, which permits unrestricted use, distribution, and reproduction in any medium, provided the original work is properly cited. 


\section{Relevant CDF detectors}

The CDF detector setup is descibed in [5] and [6]. For the results in this study we used all the CDF detectors with the exception of the time-of-flight system. The muon chambers are used only for background rejection, and silicon dectector for track quality enchancment. We will select events with just 2 Central Outer Tracker tracks, with $\sum Q=0$. We want to select events with no other hadrons produced, and will require that all the calorimetry (except around the impact points of the charged particles), the Beam Shower Counters, and the Cherenkov Luminosity Counters (CLC) have signals consistent with noise. We are therefore blind to $|\eta|>5.9$ and accept events where the proton was quasi-elastically scattered or where it fragmented into a low mass state.

\section{Rapidity gap cuts, exclusive selection}

To understand the noise levels in all the detectors, we use zero-bias (bunch crossing) triggers, taken during the same periods. We did this independently for the $1960 \mathrm{GeV}$ and $900 \mathrm{GeV}$ runs. We divided the zero-bias data into two classes: (A) no interactions, defined as no tracks, no muon stubs and no CLC hits, (B) all the other events, totally dominated by one or more inelastic interactions. Comparing the noise and signal-dominated distributions for each subdetector we determine the noise levels.

We sum the signals in each forward subdetector, and imposed cuts on the sums. This method was used in the search for exclusive $Z$-boson and observation of high mass lepton pairs [7]. In observations of exclusive $\chi_{c}$ [8] and $\gamma \gamma$ [9] we did not sum, but plotted the hottest channel and required that to be less than a cut. The methods give similar results.

The central detectors are required to be clean, except for the two charged tracks. The tracks are extrapolated to the calorimeters, and allowing any energy in a cone $\sqrt{(\Delta \eta)^{2}+(\Delta \phi)^{2}}<0.3$ around the impact points we apply the same procedure as in the forward detectors.

\section{Exclusive efficiency}

As any cross sections that we measure uses data with no other inelastic collision to spoil the exclusivity (no pile-up), we need to know the probability of having no pile-up. This is the exclusive efficiency $\varepsilon_{\text {excl }}$. The method is to look at zero-bias data, for which the luminosity of the particular bunch crossing, $L_{\text {bunch }}$, is recorded. Applying all the same cuts as before, we measure, as a function of $L_{\text {bunch }}$, the probability $P(0)$ that all the detectors are in the noise, so there was no inelastic collision (except low mass diffraction with very forward fragmentation products). The exclusive efficiency $\varepsilon_{\text {excl }}$ drops exponentially with the bunch luminosity. The slope gives the cross section for producing any hadrons in $-5.9<\eta<+5.9$. For $\sqrt{s}=1960 \mathrm{GeV}$ data the effective luminosity equals about $1.16 \mathrm{pb}^{-1}$ and the total detected inelastic cross section equals about $56.7 \mathrm{mb}$.

\section{Two exclusive tracks}

The selection of 2-track events is made with a sequence of cuts. We give higher priority to having a clean, well measured, sample than to efficiency. A big reduction comes from the central exclusivity requirement. A large part of that probably comes from expected events with charged tracks + neutrals.

The cut at $|\eta|=2.1$ is only to have a well defined boundary. The opening angle cut, removes a small number of cosmic ray tracks with $\theta_{3 D} \approx \pi$. Both tracks are required to have an small impact parameter to the nominal beam line and a small difference in $z$ projected to the beam line. We require both tracks to have a good quality. To have a well-defined fiducial region and avoid poorly known thresholds we require both tracks to have $P_{t}>0.3 \mathrm{GeV} / c$. The $P_{t}$ (pair) was required to be $<2.0 \mathrm{GeV}$. This does not reject many events, and a very small fraction of real DPE events would have such high $P_{t}$ so the background may be higher in these events. Finally we require the tracks to have opposite charge. The numbers of (++) and (- -) pairs are similar.

We are left with about $300 \mathrm{~K}$ events at $1960 \mathrm{GeV}$ and $11 \mathrm{~K}$ at $900 \mathrm{GeV}$. Most of the events have $y\left(\pi^{+} \pi^{-}\right)<1.0$. 


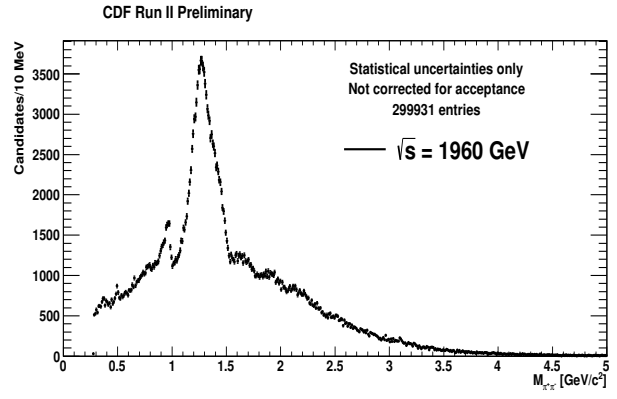

(a) $\sqrt{s}=1960 \mathrm{GeV}$

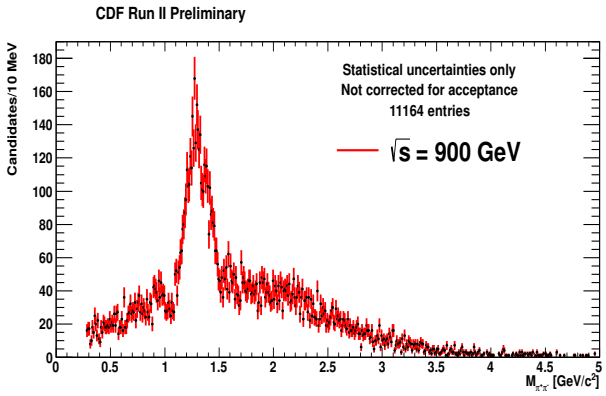

(b) $\sqrt{s}=900 \mathrm{GeV}$

Fig. 1. Invariant mass distribution of 2 particles assuming pion mass - not corrected for acceptance for two different $\sqrt{s}$.

\section{Mass distributions, resonance structures, and kinematic properties}

We now present mass spectra uncorrected for acceptance, after which we will discuss the Monte Carlo calculations of acceptance and correct to give the cross sections. As the trigger required two towers with $E_{T}>0.5 \mathrm{GeV}$, a state with $M(X) \lesssim 1 \mathrm{GeV}$ will not be accepted if it has very small $P_{t}$. So the acceptance is a strong function of both $P_{t}(X)$ and $M(X)$ when these are both small.

Table 1 shows all the states with allowed quantum numbers $I^{G} J^{P C}=0^{+}(0 / 2)^{++}$up to $1500 \mathrm{MeV}$ [10]. There are some higher broad states, mostly not well established. This data may make a valuable contribution to meson spectroscopy. States with a large gluonic content ("glueballs" or hybrids) should be favored, in contrast to $\gamma \gamma \rightarrow X$ where $q \bar{q}$ states are favored.

Fig. 1(a) and Fig. 1(b) show the mass distributions of the events in $10 \mathrm{MeV} / c^{2}$ bins, for all $P_{t}$, with statistical errors only. We have not used any particle identification, and assume here that $h^{+} h^{-}$is $\pi^{+} \pi^{-}$. This was found to be a good approximation in DPE at the CERN ISR.

Fig. 2 shows, on a log scale, the ratio of data (1960/900). The acceptance at the two energies is identical. While the ratio is consistent with being constant from threshold up to $1 \mathrm{GeV} / c^{2}$, it drops significantly in the mass region of the $f_{2}(1270)$. The lower ratio there is perhaps a spin effect. The downward trend of the ratio continues to above $4 \mathrm{GeV} / c^{2}$. The overall behavior of the $\sqrt{s}$ dependence as a function of mass gives a good benchmark to test models.

\section{$7 \pi^{+} \pi^{-}$cross sections}

In order to present cross sections, such as $d \sigma /\left(d M d P_{t} d y\right)$ and unfold acceptance $A\left(M, P_{t}, y\right)$ from the data, we calculate the acceptance, generating pion pairs and using CDF detector simulation to obtain the trigger and resonstruction efficiency. A parent state $X$ is generated, flat in rapidity with $-2.1<|y|<+2.1$, flat in mass $M(X)$ from 0 to $5.0 \mathrm{GeV} / c^{2}$, and flat in $P_{t}$ from 0 to $2.5 \mathrm{GeV} / c$. In the absence of knowledge about spins and polarizations, $X$ is made to decay isotropically ( $\mathrm{S}$-wave, $\mathrm{J}=0$ ). The acceptance $A\left(M, P_{t}\right)$ was calculated as the ratio of generated to accepted events in bins of $M(X)$ and $P_{t}(X)$.

For each $M, P_{t}$ bin we divide the data by the acceptance to get the corrected mass distribution, and use the effective luminosity to get the cross section $d \sigma / d M$, as shown in Figs. 3. Fig. 3(a) shows that the resonances structure in range $1.0-1.5 \mathrm{GeV} / \mathrm{c}^{2}$ is preserved. The mass spectra shows a broad continuum below $1 \mathrm{GeV} / c^{2}$ and a sharp drop at $1 \mathrm{GeV} / c^{2}$. These features have previously been observed at lower $\sqrt{s}$ [4]. Fig. 3(b) presents the invariant mass distribution of 2 particles assuming pion masses zoomed to the range $1.5-2.5 \mathrm{GeV} / c^{2}$. It shows an exponential drop with some bump structures. 


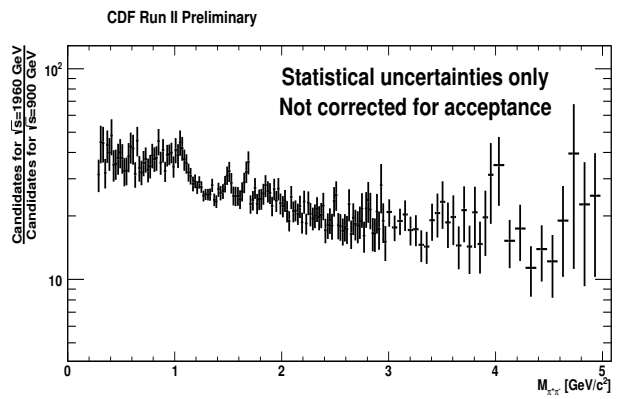

Fig. 2. Ratio of invariant mass distributions of 2 particles assuming pion mass for $\sqrt{s}=1960 \mathrm{GeV}$ and $900 \mathrm{GeV}$.

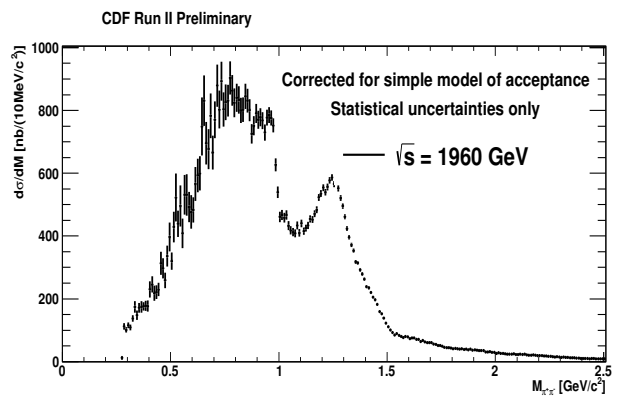

(a) $M_{\pi^{+} \pi^{-}}<2.5 \mathrm{GeV} / c^{2}$

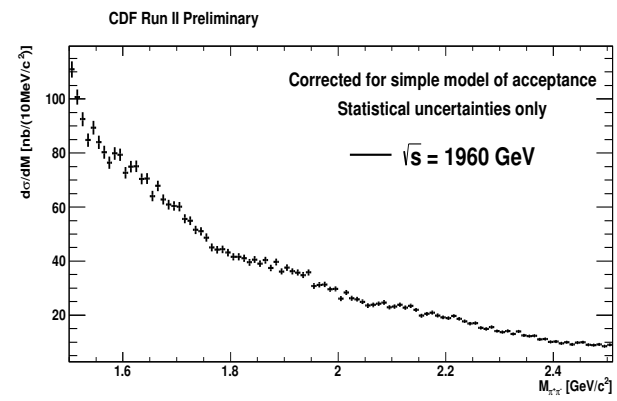

(b) $1.5 \mathrm{GeV} / c^{2}<M_{\pi^{+} \pi^{-}}<2.5 \mathrm{GeV} / c^{2}$

Fig. 3. Invariant mass distribution of 2 particles assuming pion masses - corrected for acceptance. $\sqrt{s}=1960 \mathrm{GeV}$.

Table 1. Mesons in PDG allowed in DPE. Higher mass states can be added.

\begin{tabular}{llll}
\hline Name & Mass $\left(\mathrm{MeV} / c^{2}\right)$ & Width $\left(\mathrm{MeV} / c^{2}\right)$ & B.R. $\pi^{+} \pi^{-}$ \\
\hline$f_{0}(600)$ or $\sigma$ & $400-1200$ & $250-500$ & $\sim 100 \%$ \\
$f_{0}(980)$ & $980 \pm 10$ & $40-100$ & $\sim 100 \%$ \\
$f_{2}(1270)$ & $1275.1 \pm 1.2$ & $185.1_{-2.4}^{+2.9}$ & $56.5_{-0.8}^{+1.6} \%$ \\
$f_{0}(1370)$ & $1200-1500$ & $150-250$ & seen \\
$f_{2}(1430)$ & $\sim 1430$ & $?$ & seen \\
$f_{0}(1500)$ & $1505 \pm 6$ & $109 \pm 7$ & $23.3 \pm 1.5 \%$ \\
$f_{2}^{\prime}(1525)$ & $1525 \pm 5$ & $73 \pm 6$ & $0.5 \pm 0.1 \%$ \\
\hline
\end{tabular}

\section{References}

1. J.R.Forshaw and D.A.Ross, Quantum Chromodynamics and the Pomeron, (Cambridge University Press, Cambridge, 1997)

2. V.Barone and E.Predazzi, High Energy Particle Diffraction, (Springer, Berlin Heidelberg, 2002)

3. S.Donnachie, G.Dosch, P.Landshoff and O.Nachtmann, Pomeron Physics and QCD, (Cambridge University Press, Cambridge, 2002)

4. M.G.Albrow, T.D.Coughlin, and J.R.Forshaw, Prog. Part. Nucl. Phys. 65, 149 (2010)

5. D.Acosta et al. (CDF Collaboration), Phys. Rev. D71, 032001 (2005)

6. T.Aaltonen et al. (CDF Collaboration), Phys. Rev. D77, 052004 (2008)

7. T.Aaltonen et al. (CDF Collaboration), Phys. Rev. Lett. 102, 222002 (2009)

8. T.Aaltonen et al. (CDF Collaboration), Phys. Rev. Lett. 102, 242001 (2009)

9. T.Aaltonen et al. (CDF Collaboration), Phys. Rev. Lett. 99, 242002 (2007)

10. J. Beringer et al. (Particle Data Group), Phys. Rev. D86, 010001 (2012) 